\section{The groningen longitudinal glaucoma study III. The predictive value of frequency- doubling perimetry and GDx nerve fibre analyser test results for the development of glaucomatous visual field loss}

GP Heeg ${ }^{1}$ and NM Jansonius ${ }^{1,2}$

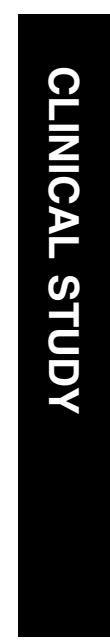

FDT and GDx; negative predictive value was 0.88 for FDT and 0.92 for GDx.

Conclusions In a clinical setting, especially GDx may be helpful for identifying glaucoma suspect patients at risk of developing glaucomatous visual field loss as assessed by SAP.

Eye (2009) 23, 1647-1652; doi:10.1038/eye.2008.348; published online 14 November 2008

Keywords: glaucoma; glaucoma suspect; FDT; GDx; scanning laser polarimetry; perimetry

\section{Introduction}

During the last decade, new devices for the diagnosis of glaucoma have been developed. Examples include the frequency-doubling perimeter (FDT; Carl Zeiss Meditec Inc., Dublin, CA, USA) and the nerve fibre analyser (GDx; Laser Diagnostic Technologies, San Diego, CA, USA). Many cross-sectional studies have shown a reasonable screening performance of these two devices. Whether they are suitable for the follow-up of glaucoma patients or whether they are able to predict glaucomatous visual field loss in glaucoma suspect patients is less clear; longitudinal studies with FDT and GDx are relatively rare. ${ }^{1-4}$

In 2000-2001, we included a cohort of consecutive patients attending our glaucoma
${ }^{1}$ Department of Ophthalmology, University Medical Center Groningen, University of Groningen, Groningen, The Netherlands

${ }^{2}$ Department of Epidemiology and Biostatistics, Erasmus Medical Center, Rotterdam, The Netherlands

Correspondence: NM Jansonius, Department of Ophthalmology, University Medical Center Groningen,

PO Box 30.001, 9700 RB Groningen, The Netherlands Tel: + 3150 3612510; Fax: + 31503611709 . E-mail: n.m.jansonius@ ohk.umcg.nl

Received: 22 April 2008 Accepted in revised form: 16 October 2008 Published online: 14 November 2008

Conflict of interest: None 
service, both patients with glaucomatous visual field loss and glaucoma suspect patients, in a prospective followup, the Groningen longitudinal glaucoma study. ${ }^{5}$ The aims of this study were to investigate (1) whether baseline FDT/GDx test results were able to predict glaucomatous visual field loss as assessed by standard automated perimetry (SAP) in glaucoma suspect patients and (2) whether FDT/GDx could be used to monitor progression in patients with glaucomatous visual field loss already present at baseline. In this study, we addressed the first aim. This issue has been addressed before for FDT and GDx separately, ${ }^{1-4}$ but, to the best of our knowledge, this is the first study investigating the combined use of FDT and GDx for this purpose.

\section{Material and methods}

The data set and other details have been described elsewhere. ${ }^{5}$ In short, all patients who visited our glaucoma service between 1 July 2000 and 30 June 2001 and who provided informed consent underwent frequency-doubling perimetry (FDT; Carl Zeiss Meditec, Dublin, California, USA), a GDx measurement (Laser Diagnostic Technologies, San Diego, California, USA), conventional perimetry (see below), and tonometry (Goldmann applanation tonometry; Haag Streit AG, Bern, Switzerland) as a baseline measurement for a prospective follow-up. The study protocol was approved by the internal review board of the department of Medical Technology Assessment of the University of Groningen. Patients received written information at home at least 2 weeks before their visit. The aim of the study was explained; participation was voluntary and participation could be stopped in any stage of the study. Additional information - if requested - was provided by a technician who was not involved in routine care and who also performed the FDT and GDx measurements (everything else was part of routine care), if the patient provided informed consent. Of the 875 (predominantly white) patients included in the study, 423 patients had normal baseline fields in both eyes. Most of these patients suffered from ocular hypertension (271; defined as $>20 \mathrm{mmHg}$ on at least two separate visits), had a positive family history of glaucoma (104; father, mother, brother, or sister) or both (61). These patients were followed irrespective of the aspect of their optic disc. Of the remaining 109 patients, 43 were followed because of a suspected optic disc as an isolated finding (defined as a vertical cup-disc ratio >0.6). Most of the remaining 66 patients had a positive family history not strictly according to our definition (grandmother, grandfather, uncle, or aunt, or a less clear history concerning father, mother, brother, or sister). All these patients, who were followed by an ophthalmologist because discharge was deemed unwise given a presumed increased risk of glaucoma, and who had normal baseline fields in both eyes, were eligible for this study. Within the framework of this study, they were named glaucoma suspect patients.

Patients were followed for a period of four years with conventional perimetry performed yearly. In the case of suspected conversion, the test was repeated to confirm or falsify the finding. Especially in the last follow-up year, the repeat test was performed shortly; all abnormal tests had to be confirmed or falsified at the end of the followup. Apparently normal but formally unreliable $\mathrm{e}^{5}$ tests were not repeated in our clinical setting. For this study, patients had to be tested with SAP (see below), both at baseline and during follow-up. Goldmann perimetry (Haag Streit AG, Bern, Switzerland) was applied in some patients (only Goldmann perimetry was available in our department until 1997 and some patients were still followed with this technique during (the beginning of) our study), but these patients were not included in the current analysis.

Glaucoma suspects who developed a reproducible visual field defect (see below) on SAP in at least one eye during follow-up were classified as converters. Patients with normal, reliable ${ }^{5}$ SAP test results in both eyes in the last follow-up year were classified as non-converters. All other patients were excluded.

\section{Perimetry}

We used the Humphrey Field analyser (HFA) 30-2 Sita Fast (Carl Zeiss Meditec Inc.). An abnormal field was defined as either

1. Glaucoma hemifield test (GHT) outside normal limits,

2. Pattern SD $(P<0.05)$, or

3. Three adjacent non-edge points $P<0.05$ in the pattern deviation probability plot of which at least one point $P<0.01$ and all points on the same side of the horizontal meridian (LTG-P criterion). ${ }^{6}$

For conversion, two consecutive, reliable ${ }^{5}$ tests had to be classified as abnormal 7,8 (according to any of the criteria as listed above), and any reliable test performed after these two tests had to be abnormal as well. Defects had to be in the same hemifield and at least partially overlapping. Moreover, defects had to be compatible with glaucoma and without any other explanation.

\section{Frequency-doubling perimetry}

Testing was performed with the FDT version 2.60, using the C-20 full threshold mode. An FDT measurement was considered positive if there was an abnormal test in at least one eye. An abnormal test was defined as $>1$ depressed 
test points $P<0.01$ in the total deviation probability plot. $^{5}$ No attention was paid to the reliability indices. ${ }^{9}$

\section{Nerve fibre analyser}

Testing was performed with the GDx version 2.010. For this study, we confined the analyses to GDx parameter 'the number'. A GDx measurement was considered positive if there was an abnormal test in at least one eye. An abnormal test was defined as the number $>29 . .^{5}$ Six images were acquired for each eye. Another six images were acquired if the first series did not yield an image with a good image quality (well-centred optic nerve head, an image in focus, equal illumination in all quadrants, and no motion artefacts). A mean image was created if there were at least two images with a good image quality.

\section{Statistical analysis}

Proportions were compared using $\chi^{2}$-statistics. The $t$-test was applied for continuous variables. Main outcome measure was the relative risk (RR) for the development of glaucomatous visual field loss of suspects with an abnormal baseline FDT/GDx test result compared to suspects with a normal baseline test result. In addition, Cox proportional hazards analyses were performed to account for follow-up duration. In converters, follow-up duration was taken until the first abnormal field that eventually resulted in the conversion classification; in non-converters, follow-up duration was taken until the end of the follow-up. A P-value of 0.05 or less was considered statistically significant.

\section{Results}

A total of 174 suspects were included in this study. Of these suspects, 134 suffered from ocular hypertension, 49 had a positive family history of glaucoma, 53 had a vertical cup-disc ratio $>0.6$, and 163 had at least one of these three characteristics. The remaining 11 suspects had at least two less strict characteristics (an IOP at least twice $\geqslant 20 \mathrm{mmHg}$, a less strict positive family history (see Materials and methods), or a vertical cup-disc ratio equal to 0.6 ). Table 1 shows the characteristics of the included suspects. Of the excluded suspects, $115 \mathrm{did}$ not visit our department during the last year of the follow-up, $72 \mathrm{had}$ a visit without an HFA test (Goldmann test or no test at all), and 62 had ambiguous test results (mainly normal test results that had to be classified as unreliable but were - in our clinical setting - not repeated within the same year because of a low suspicion of abnormality).

Of the included suspects, 26 of 174 were classified as converters. This corresponds to a conversion rate of $3.7 \%$
Table 1 Characteristics of the included glaucoma suspect patients $(n=174)$ : age at baseline, peak intraocular pressure (the highest IOP ever measured), intraocular pressure at baseline, sex, family history of glaucoma, myopia, IOP-lowering treatment at any time during follow-up, and vertical cup-disc ratio at baseline

\begin{tabular}{|c|c|}
\hline Age (years) ${ }^{a}$ & $60(13)$ \\
\hline Peak IOP $(\mathrm{mmHg})^{\mathrm{a}, \mathrm{b}}$ & $27(6)$ \\
\hline IOP at baseline $(\mathrm{mmHg})^{\mathrm{a}, \mathrm{b}}$ & $19(4)$ \\
\hline Sex (\% male $)$ & 46 \\
\hline Family history (\%) & 28 \\
\hline Myopia (\%) & 18 \\
\hline Treatment IOP (\%) & 45 \\
\hline Vertical cup-disc ratio $>0.6(\%)^{\mathrm{b}}$ & 39 \\
\hline
\end{tabular}

${ }^{a}$ Mean (SD)

${ }^{\mathrm{b}}$ Higher value of both eyes.

per year (95\% confidence interval (CI): $2.4-5.1 \%$ per year). In the subgroup of patients with ocular hypertension without a positive family history of glaucoma, this rate was 3.8\% per year; in the subgroup of patients with a positive family history of glaucoma without ocular hypertension, this rate was $2.2 \%$ per year $(P=0.58)$. Of the remaining 148 non-converters, 77 had a normal HFA during all follow-up visits, whereas 71 had one or more abnormal fields during follow-up (but their last field was - by definition - normal). Table 2 presents the characteristics of the converters and non-converters. On average, there was no significant difference between the two groups, except for the vertical cup-disc ratio at baseline.

Table 3 shows the baseline FDT and GDx test results of converters and non-converters. The RR for conversion of an abnormal baseline FDT was 1.8 (CI: $0.9-3.7 ; P=0.10)$ and of an abnormal baseline GDx 2.7 (CI: 1.2-6.3; $P=0.01)$. Positive predictive value was 0.22 for both FDT $(11 /(11+39))$ and GDx $(19 /(19+68))$, to be compared to the prior probability of conversion, being 0.15 (26/174; CI: $0.10-0.20)$. Negative predictive value was 0.88 for FDT $(109 /(15+109))$ and 0.92 for GDx $(80 /(7+80))$, to be compared to the prior probability of no conversion, being 0.85 (148/174; CI: 0.80-0.90). Similar results were found using Cox proportional hazards analysis, taking follow-up duration into account (FDT: hazard ratio 1.9 (CI: $0.9-4.1 ; P=0.10)$; GDx: hazard ratio 2.8

(CI: $1.2-6.7 ; P=0.02)$ ).

Table 4 shows the combined use of FDT and GDx. As mentioned above, 15\% (26 of 174) of the suspects converted during the follow-up. Of those with a normal baseline GDx, only $8 \%$ (7 of 87 ) converted. In these patients, the baseline FDT apparently did not yield any additional information. Of those with an abnormal baseline GDx, 22\% (19 of 87) converted. In these patients, the baseline FDT seemed to be more informative: of the patients with an abnormal baseline FDT, 30\% converted, 
Table 2 Characteristics of converters $(n=26)$ and nonconverters $(n=148)$

\begin{tabular}{|c|c|c|c|}
\hline & Converters & Non-converters & P-value \\
\hline Age (years) ${ }^{a}$ & $64(10)$ & $60(13)$ & 0.12 \\
\hline Peak IOP $(\mathrm{mmHg})^{\mathrm{a}, \mathrm{b}}$ & $26(5)$ & $27(6)$ & 0.37 \\
\hline IOP at baseline $(\mathrm{mmHg})^{a, b}$ & $19(4)$ & $19(4)$ & 0.83 \\
\hline Sex ( $\%$ male $)$ & 40 & 47 & 0.66 \\
\hline Family history (\%) & 22 & 29 & 0.63 \\
\hline Myopia (\%) & 25 & 17 & 0.50 \\
\hline Treatment IOP (\%) & 40 & 46 & 0.73 \\
\hline $\begin{array}{l}\text { Vertical cup-disc ratio } \\
>0.6(\%)^{\mathrm{b}}\end{array}$ & 62 & 35 & 0.009 \\
\hline
\end{tabular}

For details see legend to Table 1 .

${ }^{\mathrm{a}}$ Mean (SD).

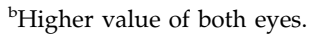

Table 3 Baseline FDT and GDx test results for converters and non-converters

\begin{tabular}{lcccc}
\hline & $T D<2$ & $T D>1$ & $n<30$ & $n>29$ \\
\hline Converters & 15 & 11 & 7 & 19 \\
Non-converters & 109 & 39 & 80 & 68 \\
\hline
\end{tabular}

$\mathrm{TD}=$ number of depressed frequency doubling perimetry test points $P<0.01$ in the total deviation probability plot, $N=$ nerve fibre analyser parameter The Number.

Table 4 Combined use of FDT and GDx

\begin{tabular}{lcc}
\hline GDx & FDT & Percentage converters \\
\hline- & - & $9(6$ of 70$)$ \\
- & + & $6(1$ of 17$)$ \\
+ & - & $17(9$ of 54$)$ \\
+ & + & $30(10$ of 33$)$ \\
\hline$-=$ normal baseline test result; $+=$ abnormal baseline test result.
\end{tabular}

as compared to $17 \%$ of the patients with a normal baseline FDT. The small numbers of converters in the various rows of Table 4, however, do not warrant firm conclusions. In line with these findings, Cox proportional hazards analysis applied to FDT and GDx simultaneously revealed an at most modest additional yield of FDT in the case of a combined use of both techniques (FDT: hazard ratio: 1.6 (CI: $0.7-3.5 ; P=0.26$ ); GDx: hazard ratio: 2.6 (CI: 1.1-6.2; $P=0.04)$ ).

None of the converters developed serious glaucomatous damage during the 4 years of follow-up. At the end of the follow-up, the mean deviation (MD) of the HFA of the worse eye of the converters ranged from -7.6 to $-0.8 \mathrm{~dB}$, with an average value of $-3.6 \mathrm{~dB}$.

\section{Discussion}

This study describes the outcome of a large cohort of glaucoma suspect patients who were followed prospectively in an observational study, the Groningen longitudinal glaucoma study. We found an increased risk of developing glaucomatous visual field loss in suspects with an abnormal baseline GDx.

Landers $e t \mathrm{al}^{1}$ followed 62 suspects prospectively for three years. At the end of the follow-up, five patients had converted to abnormal SAP (conversion rate: $2.7 \%$ per year), all of whom had an abnormal baseline FDT. Medeiros $e t a l^{2}$ followed 105 suspects for 3.4 years. They found conversion to abnormal SAP in 17 patients (4.7\% per year) and found an RR for conversion to glaucoma of an abnormal baseline FDT of 3.2 (CI: 1.2-8.3). Mohammadi et $\mathrm{al}^{3}$ followed 160 suspects for 3.8 years. They found conversion to abnormal SAP in 16 patients (2.6\% per year) and found several GDx parameters to be significant predictors of conversion. Kamantigue $e t \mathrm{al}^{4}$ followed 63 eyes of 50 suspects for 62 months. They found conversion to abnormal SAP in $14 \%$ of the eyes (2.8\% per year) and found an abnormal baseline FDT to be a significant predictor. Katz et $a l^{7}$ and Johnson $e t a l^{8}$ found conversion rates of 2.8 and $4.4 \%$ per year, respectively. Our conversion rate $(3.7 \%$ per year) and RR findings (1.8 for FDT; 2.7 for GDx) are in line with those earlier studies. As compared to the ocular hypertension treatment study, those conversion rates are somewhat high. ${ }^{10}$ A possible explanation for this difference could be that patients in the ocular hypertension treatment study were entered into the study in an - on average - earlier stage of the disease. ${ }^{11}$

An RR should always be interpreted together with a prior probability. In the presence of a low prior probability of conversion, the clinical significance of a statistically significant RR of 2.7 , as found for GDx, is limited. In our study, the prior probability of conversion was $0.15(26 / 174)$ after a 4-year follow-up. With an abnormal GDx, the probability of conversion increased from 0.15 to 0.22 (positive predictive value); with a normal GDx, the probability of no conversion increased from 0.85 (1-0.15) to 0.92 (negative predictive value).

There is an ongoing discussion as to whether FDT and GDx are able to detect glaucoma earlier than SAP. This study, and also the earlier studies, ${ }^{1-4}$ showed prospectively that in some cases FDT and GDx are able to detect abnormalities earlier than SAP. However, these studies are not suitable to settle this discussion, as the cases that were included were required to have normal SAP test results at baseline, yielding a selection bias towards negative SAP findings. Several cross-sectional studies have revealed suboptimal FDT and GDx sensitivity for early glaucoma, that is, cases with abnormal SAP, but normal FDT/GDx are not uncommon. ${ }^{5,12,13}$ Apparently, some cases are detected by FDT/GDx before SAP, whereas other cases are detected by SAP first. Longitudinal studies starting with normal 
baseline test results for all tests should settle this discussion. ${ }^{14}$ Likewise, the fact that abnormal GDx findings $(87 / 174)$ were more common at baseline than abnormal FDT findings (50/174; using cutoff points that yielded similar specificities with both techniques ${ }^{5}$ ) might suggest that structural changes precede functional changes. The fact that the suspects in our study were classified by requiring a normal functional test (HFA), however, might have induced a bias towards normal functional test findings.

For the cutoff points as used in this study, FDT and GDx have similar sensitivities and specificities (0.90 and 0.81 for FDT; 0.90 and 0.78 for GDx). ${ }^{5}$ At this cutoff point, FDT predicted only 11 of the 26 converters, whereas GDx predicted 19 (Table 3). To increase the number of converters predicted by FDT, the cutoff point could be lowered to $>0$. With this cutoff point, 70 of the 174 participants would have had an abnormal baseline test result and 16 of the 26 converters would have been predicted by FDT (RR: 2.4 (1.1-5.0)). At this cutoff point, the sensitivity and specificity of FDT were 0.94 and $0.70 .{ }^{5}$ For GDx, these sensitivity and specificity values can be reached with a cutoff point $>25$. With this cutoff point, 101 of the 174 participants would have had an abnormal baseline GDx and 21 of the 26 converters would have been predicted by GDx (RR: 3.0 (1.2-7.6)).

The vast majority of the converters were within a range often denoted as early glaucoma (HFA MD: $-6 \mathrm{~dB}$ or more) at the end of the follow-up; none of the converters had developed severe glaucomatous damage in either eye. This finding reflects the slow nature of glaucoma, and together with the low conversion rate, it is clear that visits of glaucoma suspects intended to screen for glaucoma may generally be widely spaced in time. Some delay in detecting an incident glaucoma case will not immediately cause visual impairment, and frequent visits will induce too many false-positive findings because of the low prior probability of glaucoma (prior probability $=$ conversion rate $\times$ intertest interval).

The Groningen longitudinal glaucoma study is a purely observational study, that is, all the data were collected as part of routine clinical care. IOP and HFA data were available for the clinician and thus could, for example, have influenced the decision to start or stop IOP-lowering medication. An exception was made for the FDT and GDx measurements. These were performed by a separate technician and were not available for the clinician. The aim of - and the philosophy behind - the study was that it should yield information concerning the additional value of FDT and GDx measurements in a clinical setting.

All analyses in this study were performed on a 'by patient' basis. A glaucoma patient is a patient with glaucoma in at least one eye, a suspect is a patient with normal fields in both eyes. Likewise, an FDT/GDx test result is normal if normal in both eyes, and abnormal if abnormal in at least one eye. We did not require that SAP defects had to develop in the same area or eye as predicted by baseline FDT/GDx abnormalities. After all, if a test correctly identifies a patient as having glaucoma, or as being at risk of getting glaucoma, by showing an abnormality anywhere, it is a good test. For confirmation of SAP conversion itself, however, we required - as mentioned in the Materials and methods section - topographical consistency.

Unlike SAP abnormalities, baseline FDT/GDx abnormalities were not confirmed or falsified. In this study, baseline FDT/GDx were considered determinants and SAP conversion was the dependent variable. Some misclassification of determinants results in a conservative risk estimate. Hence, our conclusion that GDx can predict SAP conversion will not be affected by the fact that we only had a single baseline test. In FDT, on the other hand, misclassification at baseline could have masked a significant result. A learning effect was studied separately, but no learning effect was found. ${ }^{15}$ In line with this finding, of the 50 patients with an abnormal FDT at baseline, only seven had a normal test result during the first follow-up visit. Patients with an apparently normal but formally unreliable HFA test result in the last follow-up year were excluded. This might be a possible source of bias.

It is an intrinsic problem of longitudinal studies that devices (or preferred treatment modalities) may change during the study. A 24-2 version of FDT (Matrix) has been developed with 54 test locations instead of 17, and a version of GDx with a variable corneal compensator (GDx-VCC) has been launched. Neither the Matrix nor the GDx-VCC was available at the time we started our study. Shortly after the introduction of the SITA strategies, SITA fast was adopted in our department. Change of strategy during a longitudinal study was deemed unwise. Two studies reported a slightly higher sensitivity for SITA standard in comparison with SITA fast; ${ }^{16,17}$ one study reported a higher sensitivity for SITA fast. ${ }^{18}$ The highest reported sensitivity difference was $7 \%$ (based on a group of only 26 patients). ${ }^{17}$ Hence, we would have detected at most two more converters by using SITA standard instead of SITA fast.

In conclusion, in a clinical setting, abnormal GDx findings in glaucoma suspect patients are related to an increased risk of developing glaucomatous visual field loss. GDx may, therefore, be helpful for the clinician to optimise the care for this group of patients. Especially in the case of normal findings, the next screening visit may be postponed, and in the case of borderline IOP readings, the start of IOP-lowering treatment may be delayed. For FDT, the findings were less pronounced. 


\section{Acknowledgements}

This study was supported by the Dutch Health Care Insurance Council (CVZ) and the University Medical Centre Groningen, the Netherlands.

\section{References}

1 Landers JA, Goldberg I, Graham SL. Detection of early visual field loss in glaucoma using frequency-doubling perimetry and short-wavelength automated perimetry. Arch Ophthalmol 2003; 121: 1705-1710.

2 Medeiros FA, Sample PA, Weinreb RN. Frequency doubling technology perimetry abnormalities as predictors of glaucomatous visual field loss. Am J Ophthalmol 2004; 137: 863-871.

3 Mohammadi K, Bowd C, Weinreb RN, Medeiros FA, Sample PA, Zangwill LM. Retinal nerve fiber layer thickness measurements with scanning laser polarimetry predict glaucomatous visual field loss. Am J Ophthalmol 2004; 138: 592-601.

4 Kamantigue ME, Joson PJ, Chen PP. Prediction of visual field defects on standard automated perimetry by screening C20-1 frequency doubling technology perimetry. J Glaucoma 2006; 15: 35-39.

5 Heeg GP, Blanksma LJ, Hardus PLLJ, Jansonius NM. The Groningen Longitudinal Glaucoma Study. I. Baseline sensitivity and specificity of the frequency doubling perimeter and the GDx nerve fibre analyser. Acta Ophthalmol Scand 2005; 83: 46-52.

6 Katz J, Sommer A, Gaasterland DE, Anderson DR. Comparison of analytic algorithms for detecting glaucomatous visual field loss. Arch Ophthalmol 1991; 109: 1684-1689.

7 Katz J, Quigley HA, Sommer A. Detection of incident field loss using the glaucoma hemifield test. Ophthalmology 1996; 103: 657-663.

8 Johnson CA, Sample PA, Cioffi GA, Liebmann JR, Weinreb RN. Structure and function evaluation (SAFE): I. Criteria for glaucomatous visual field loss using standard automated perimetry (SAP) and short wavelength automated perimetry (SWAP). Am J Ophthalmol 2002; 134: 177-185.
9 Heeg GP, Jansonius NM. An apparently poor test reliability, as assessed by reliability indices, does not compromise the screening performance of frequency doubling perimetry. Am J Ophthalmol 2006; 141: 585-587.

10 Kass MA, Heuer DK, Higginbotham EJ, Johnson CA, Keltner JL, Miller JP et al. The Ocular Hypertension Treatment Study: a randomized trial determines that topical ocular hypotensive medication delays or prevents the onset of primary open-angle glaucoma. Arch Ophthalmol 2002; 120: 701-713.

11 Shah AR, Del Priore LV. Progressive visual field loss in subfoveal exudation in age-related macular degeneration: a meta-analysis using lineweaver-burke plots. $A m \mathrm{~J}$ Ophthalmol 2007; 143: 83-89.

12 Cello KE, Nelson-Quigg JM, Johnson CA. Frequency doubling technology perimetry for detection of glaucomatous visual field loss. Am J Ophthalmol 2000; 129: 314-322.

13 Trible JR, Schultz RO, Robinson JC, Rothe TL. Accuracy of glaucoma detection with frequency-doubling perimetry. Am J Ophthalmol 2000; 129: 740-745.

14 Jansonius NM, Heeg GP. The Groningen Longitudinal Glaucoma Study. II. A prospective comparison of frequency doubling perimetry, the GDx nerve fibre analyser and standard automated perimetry in glaucoma suspect patients. Acta Ophthalmol Scand, July 2008 [e-pub ahead of print].

15 Heeg GP, Ponsioen TL, Jansonius NM. Learning effect, normal range, and test-retest variability of frequency doubling perimetry as a function of age, perimetric experience, and the presence or absence of glaucoma. Ophthalmol Physiol Opt 2003; 23: 535-540.

16 Delgado MF, Nguyen NT, Cox TA, Singh K, Lee DA, Dueker DK et al. Automated perimetry: a report by the American Academy of Ophthalmology. Ophthalmology 2002; 109: 2362-2374.

17 Budenz DL, Rhee P, Feuer WJ, McSoley J, Johnson CA, Anderson DR. Sensitivity and specificity of the Swedish interactive threshold algorithm for glaucomatous visual field defects. Ophthalmology 2002; 109: 1052-1058.

18 Pierre-Filho PT, Schimiti RB, de Vasconcellos JP, Costa VP. Sensitivity and specificity of frequency-doubling technology, tendency-oriented perimetry, SITA standard and SITA fast perimetry in perimetrically inexperienced individuals. Acta Ophthalmol Scand 2006; 84: 345-350. 